\title{
Predictive Ability of Preoperative CT Scan in Determining Whether the Adrenal Gland is Spared at Radical Nephrectomy
}

\author{
Gregory J. Nason Asadullah Aslam Subhasis K. Giri \\ Department of Urology, University Hospital Limerick, Limerick, Ireland
}

\author{
Key Words \\ Adrenal gland $\cdot$ Cancer detection $•$ Renal tumor • \\ Computed tomography $\cdot$ Oncologic surgery • \\ Kidney tumor $\cdot$ Organ confined disease
}

\begin{abstract}
Introduction: The aim of this study was to assess whether preoperative multiple detector computed tomography (MDCT) accurately predicts adrenal involvement for patients undergoing non-adrenal sparing radical nephrectomy for renal cell carcinoma. Methods and Materials: A retrospective observational study based on a composite patient population of two university teaching hospitals who underwent radical nephrectomy. Sensitivity, specificity, overall accuracy, positive and negative predictive values and likelihood ratios were calculated from radiological reports. Results: Total 579 patients underwent radical nephrectomy, of which 199 (34.4\%) patients underwent a non-adrenal sparing radical nephrectomy, in which 128 (64.3\%) were male and 118 (59.3\%) were left side tumors. Mean tumor size was $8.2 \mathrm{~cm}$ (range $1.4-20 \mathrm{~cm}$ ). MDCT was found to have a sensitivity of $100 \%$ and specificity of $95.2 \%$ for identifying adrenal invasion. Total 179 patients (89.9\%) had a radiographically normal ipsilateral adrenal gland, of which none were found to have adrenal involvement. Therefore, the negative predictive value of preoperative cross-sectional imaging for identification of adrenal involvement was $100 \%$. Conclusion: Cross- sectional MDCT imaging accurately predicts adrenal involvement and the decision to remove or spare the adre-
\end{abstract}

\section{KARGER}

Fax +4161306 1234

E-Mail karger@karger.ch

www.karger.com
(C) 2015 S. Karger AG, Basel

1015-9770/15/0093-0143\$38.00/0

Accessible online at:

www.karger.com/cur nal gland should be made preoperative planning regardless of tumour size or location at the time of multi-disciplinary discussion unless there is intraoperative evidence of adrenal invasion.

Copyright @ 2015 S. Karger AG, Basel

\section{Introduction}

Radical nephrectomy $(\mathrm{RN})$ is the treatment of choice for early stage renal cell carcinoma (RCC) where partial nephrectomy is not safe or feasible. Ipsilateral adrenalectomy (IA) at the time of $\mathrm{RN}$ was considered crucial for cancer control and thus was routine from the time of its initial description by Robson et al in 1969 [1].

The availability of modern cross sectional imaging such as multiple detector computed tomography (MDCT) has resulted in not only an earlier presentation and detection of lower stage renal tumors $[2,3]$ but also accurate characterisation of adrenal gland involvement by RCC [4].

The current European Association of Urology guidelines for RCC state that IA during RN has no survival advantage and is not recommended when there is no clinical evidence of invasion of the adrenal gland [5]. Ipsilateral adrenal invasion is rare about $1-5 \%[6,7]$. In a systematic review of adrenalectomy at the time of $\mathrm{RN}$, O'Malley et al., concluded IA should be considered in select cases in which there are risk factors for adrenal in- 
Table 1. Patient demographics and clinico-pathological characteristics

\begin{tabular}{ll}
\hline Classification & Value \\
\hline Mean Age & 59.6 years old \\
Male $(\%)$ & $128(64.3)$ \\
Tumor size (cm) & \\
Mean (range) & $8.2(1.4-20)$ \\
<4 cm & $26(13.1 \%)$ \\
4-7cm & $54(27.1 \%)$ \\
> 7cm & $119(59.8 \%)$ \\
Tumor Location & \\
Upper pole & $143(71.9 \%)$ \\
Lower pole & $32(16.1 \%)$ \\
Inter-polar & $24(12.1 \%)$ \\
Side & \\
Left & $118(59.3 \%)$ \\
Right & $81(40.7 \%)$ \\
T-stage & \\
T1a & $21(10.6 \%)$ \\
T1b & $31(15.6 \%)$ \\
T2 & $34(17.1 \%)$ \\
T3a & $52(26.1 \%)$ \\
T3b & $56(28.1 \%)$ \\
T4 & $5(2.5 \%)$ \\
Histological Subtype & \\
Clear Cell & $172(86.4 \%)$ \\
Papillary & $13(6.5 \%)$ \\
Chromophobe & $11(5.5 \%)$ \\
Other & $3(1.5 \%)$ \\
Fuhrman Grade & \\
G1 & $9(4.5 \%)$ \\
G2 & $78(39.2 \%)$ \\
G3 & $77(38.7 \%)$ \\
IV4 invasion & $35(17.6 \%)$ \\
Tumor necrosis & $7(3.5 \%)$ \\
Adrenal invasion & $80(40.2 \%)$ \\
& $11(5.5 \%)$ \\
&
\end{tabular}

volvement [8]. Despite this, there remains wide variation in practice regarding the removal or preservation of the adrenal gland at RN. Yap et al. [9, 10], reported a 30\% adrenalectomy rate for small organ confined RCC with an associated worse overall survival and this may be due broad clinical indication of adrenalectomy during $\mathrm{RN}$ as suggested in the EAU guidelines. Thus there is need for better preoperative planning with regard to the decision to remove or preserve the adrenal gland.

The aim of this study was to assess whether preoperative MDCT accurately predicts adrenal involvement for patients undergoing non-adrenal sparing radical nephrectomy (NASRN) for RCC.
Table 2. Tumour characteristics with or without adrenal invasion

\begin{tabular}{llll}
\hline & Adrenal Invasion & No adrenal invasion & $\mathrm{p}$ \\
\hline $\mathrm{n}$ & 11 & 188 & \\
Size (range) cm & $9.9(5.5-20)$ & $8.1(1.4-20)$ & 0.088 \\
Fuhrman G3/4 (\%) & $11(100 \%)$ & $101(53.7 \%)$ & 0.003 \\
Upper Pole & $8(72.3 \%)$ & $110(58.5 \%)$ & 0.530 \\
IVC invasion & $1(9.1 \%)$ & $6(3.2 \%)$ & 0.333 \\
Tumor necrosis & $5(45.5 \%)$ & $75(39.9 \%)$ & 0.758 \\
\hline
\end{tabular}

\section{Methods and Materials}

This was a retrospective observational study based on a composite patient population of two university teaching hospitals who underwent radical nephrectomy for RCC between January 2000 and December 2012 following review by the institutional review board. Demographic, clinical and pathological (tumor, node, metastases classification) details on these patients were collected.

Histopathological data were obtained from the final pathology. Tumors were graded according to the Fuhrman grading system for RCC. Pathological details were assessed from final histopathological reports. The tumor, node, metastases classification was adjusted according to the American Joint Committee on Cancer 2010 guidelines. All histological specimens were independently reviewed by at least 2 consultant histopathologists. All cases were discussed pre and post operatively at departmental multi-disciplinary meetings before and after RN. Our exclusion criteria included patients who underwent an adrenal sparing radical nephrectomy (ASRN), benign histology and those with transitional cell carcinoma. All patients had tri-phasic renal protocol MDCT as part of staging investigation. Tumor location was identified based on review of preoperative radiology imaging. Radiological involvement of the adrenal gland was considered when the gland appeared enlarged, nodular and irregular in outline or contained a soft tissue lesion not consistent with adenoma by radiographic criteria (less than 10 Hounsfield units on unenhanced computed tomography) or was obscured by the renal tumor mass.

Diagnostic accuracy of MDCT scan (designed to distinguish involved adrenal gland from uninvolved adrenal gland) can be quantified by the measures such as sensitivity and specificity, overall accuracy, positive and negative predictive values and positive and negative likelihood ratios.

Statistical analysis was performed using Pearson's $\chi^{2}$ test and the Fisher exact test to compare categorical variables, and the Student $t$-test for comparison of continuous variables (SPSS Version 21.0 New York, USA). A p-value of 0.05 was considered statistically significant.

\section{Results}

Between 2000 and 2012, 579 patients underwent RN, of which 199 (34.4\%) patients underwent a NASRN. The mean age of these patients was 59.6 years and 128 
Table 3. Sensitivity, specificity, positive predictive value, negative predictive value of cross sectional imaging in identifying adrenal invasion

\begin{tabular}{llll}
$\begin{array}{l}\text { Adren index test } \\
\text { (CT imaging) }\end{array}$ & \multicolumn{3}{c}{ Reference standard (Histopathology) } \\
\cline { 2 - 4 } Adrenal Invasion (+) & No adrenal invasion (-) & Total \\
Imaging (+) & $11(\mathrm{TP})$ & $9(\mathrm{FP})$ & 20 \\
Imaging (-) & $0(\mathrm{FN})$ & $179(\mathrm{TN})$ & 179 \\
Total & 11 & 188 & 199 \\
\hline
\end{tabular}

$\mathrm{TP}=$ True positive $\mathrm{FN}=$ false negative $; \mathrm{FP}=$ false positive $; \mathrm{TN}=$ true negative.

Table 4. Sensitivity, specificity, overall accuracy, positive predictive value, negative predictive value, positive likelihood ratio and negative likelihood ratio of cross sectional CT imaging in identifying adrenal invasion

\begin{tabular}{lc}
\hline \multicolumn{1}{c}{ CT performance characteristics for } & Percentage \\
\hline Sensitivity & 100 \\
Specificity & 95.2 \\
Overall accuracy & 95.4 \\
Positive predictive value & 55 \\
Negative predictive value & 100 \\
Positive likelihood ratio & 23.8 \\
Negative likelihood ratio & 0 \\
\hline
\end{tabular}

$(64.3 \%)$ were male. Mean tumor size was $8.2 \mathrm{~cm}$ (range $1.4-20 \mathrm{~cm})$.

Histopathological characteristics are recorded in table 1 . There was evidence of adrenal involvement in 11 $(5.5 \%)$ patients.

Patients who had evidence of adrenal invasion had significantly higher grade tumors- Fuhrman G3/4 (100 vs. $53.7 \%, p=0.003$ ). Tumor size, location, evidence of vena-caval invasion or tumor necrosis was not associated with any difference (table 2).

MDCT images and official radiology reports were reviewed for all patients. MDCT was found to have a sensitivity of $100 \%$ and specificity of $95.2 \%$ for identifying adrenal invasion (table 3 ).

Overall, 179 patients $(89.9 \%)$ had a radiographically normal ipsilateral adrenal gland, of which none were found to have adrenal involvement. Therefore, the negative predictive value of preoperative cross-sectional imaging for identification of adrenal involvement was $100 \%$. Table 4 demonstrates very high predictive ability of CT imaging with regard to involvement of adrenal gland.

Pre-Operative CT Determines Fate if Adrenal Gland is Spared
Of the 11 patients with histopathological confirmed adrenal invasion- all of these patients had evidence of direct adrenal invasion on CT. Eight (72.3\%) had upper pole tumors. One patient had distant metastatic disease at presentation with lung metastases. There was no history of bilateral renal tumors.

Of the nine patients who had radiological evidence of adrenal invasion but no histopathological evidence of invasion, the imaging was re-examined by an independent radiologist. In each case- the independent radiologist gave a similar report of adrenal invasion- in each case there was loss or distortion of the fat plane between the kidney and the adrenal with an upper pole tumor.

\section{Discussion}

Our study demonstrates that cross sectional MDCT imaging accurately out rules adrenal invasion with RCC. In our cohort, adrenal invasion was present in only $5.5 \%$. All of those patients had suspicion of adrenal involvement on CT. Histologically we did not identify adrenal involvement in any patient with a negative preoperative imaging. Thus, an ASRN can be safely planned preoperatively based on MDCT imaging regardless of tumour size or location. This study echoes the findings of Blakely et al. [12], who also showed $100 \%$ negative predictive value of modern cross-sectional imaging with regard to adrenal involvement.

There has been a considerable shift towards partial nephrectomy and the current EAU guidelines recommend nephron sparing surgery should be performed irrespective of surgical approach [5]. Despite this, RN is still often performed for organ confined RCC where nephron sparing surgery is not safe or feasible. Although IA was an element of RN at the time of its initial description (Robson's radical nephroadrenalectomy [1]) - there is

Curr Urol 2015;9:143-147 
evidence and guidelines against this routine practice [5, $8,12]$. Two systematic reviews do not support the practice of IA at RN. Bekema et al. [13] failed to demonstrate any oncological benefit of concomitant adrenalectomy for locally advanced RCC. O'Malley et al. [8] demonstrated similar overall and disease-specific survival in patients undergoing $\mathrm{RN}$ with or without adrenalectomy. Furthermore, for partial nephrectomy, even for upper pole tumors, adrenalectomy should not be routinely performed [14].

Overall the incidence of adrenal invasion is low (< $5 \%$ ), similar to our findings. Certain tumour characteristics have traditionally been associated with an increased risk of adrenal involvement- such as tumor size, tumor location, venous thrombus status, and radiographic appearance have all been proposed as factors in decision making [6]. Recent evidence has suggested a shift from this practice. Kutikov et al. [7] reported high sensitivity and specificity of radiological imaging in identifying adrenal invasion, similar to our findings. Pre-operative imaging should inform the decision to preserve or remove the adrenal gland as opposed to tumor site or size.

However IA is still being performed in some settings despite its low incidence and lack of evidence supporting significant oncological benefits. Yap et al. [9] reported a series of pT1a $(<4 \mathrm{~cm})$ renal cell carcinoma that underwent radical nephrectomies from the Ontario Cancer Registry. The overall ipsilateral adrenalectomy rate was $30 \%$ with an associated reduced overall 10 year survival rate ( 79.8 vs. $74.1 \%$, adrenalectomy versus adrenal-sparing). However, there was no difference in cancer specific survival (94.5 vs. 93.3\%), adrenalectomy versus adrenal-sparing) which was expected given the excellent prognosis with pT1a tumors. Bazzi et al. [15] reported a $48 \%$ adrenalectomy rate in Memorial Sloan-Kettering in 802 patients undergoing surgery for locally advanced RCC, although adrenalectomy rates decreased with time. While Blakely et al. [12] reported a $40 \%$ IA rate despite only 5\% having adrenal involvement in a series of 117 patients.

Apart from a lack of evidence demonstrating any survival benefit of a NASRN, there are numerous reason to promote the preservation of the adrenal gland. Although IA is not associated with significant Intraoperative morbidity, there are possible long-term effects associated with a solitary adrenal gland. The risk of developing an ipsilateral or contralateral adrenal metastasis is independent of IA at the time of $\mathrm{RN}$ even in high risk tumors [6]. Furthermore, there have been reports of impairment of adreno-cortical function even with a remaining function- ing contralateral adrenal gland $[16,17]$.

We acknowledge our retrospective cohort study with obvious limitations. Firstly our institution is a tertiary referral centre and the imaging studies were not all performed or reported centrally. Despite this, all studies were reviewed at the institutional multi-disciplinary meeting preoperatively. Furthermore, despite this being a historical series, the histological specimens were not re-examined and updated by a consultant histopathologists. However this does improve the generalisability of our data.

\section{Conclusion}

Ipsilateral adrenal invasion by RCC is rare. Crosssectional MDCT imaging accurately predicts adrenal involvement and the decision to remove or spare the adrenal gland should be made preoperative planning regardless of tumour size or location at the time of multi-disciplinary discussion unless there is intraoperative evidence of adrenal invasion. 


\section{References}

1 Robson CJ, Churchill BM, Anderson W: The results of radical nephrectomy for renal cell carcinoma. J Urol 1969;101:297-201.

2 Kane CJ, Mallin K, Ritchey J, Cooperberg MR, Carroll PR: Renal cell cancer stage migration: analysis of the National Cancer Data Base. Cancer 2008;113:78-83.

3 Looney AT, Nason GJ, McGuire BB, Kelly ME, Mulvin DW, Galvin DJ, Quinlan DM, Lennon GM: Incidentalology: a developing urological sub-specialty. Surgeon 2014;12: 301-306.

4 Gill IS, McClennan BL, Kerbl K, Carbone JM, Wick M, Clayman RV: Adrenal involvement from renal cell carcinoma: predictive value of computerized tomography. J Urol 1994;152:1082-1085.

5 Ljungberg B, Bensalah K, Canfield S, Dabestani S, Hofmann F, Hora M, Kuczyk MA, Lam T, Marconi L, Merseburger AS, Mulders P, Powles T, Staehler M, Volpe A, Bex A: EAU guidelines on renal cell carcinoma: 2014 Update. Eur Urol 2015;67:913-924.

6 Weight CJ, Kim SP, Lohse CM, Cheville JC, Thompson RH, Boorjian SA, Leibovich BC: Routine adrenalectomy in patients with locally advanced renal cell cancer does not offer oncologic benefit and places a significant portion of patients at risk for an asynchronous metastasis in a solitary adrenal gland. Eur Urol 2011;60:458-464.
7 Kutikov A, Piotrowski ZJ, Canter DJ, Li T, Chen DY, Viterbo R, Greenberg RE, Boorjian SA, Uzzo RG: Routine adrenalectomy is unnecessary during surgery for large and/or upper pole renal tumors when the adrenal gland is radio- graphically normal. J Urol 2011; 185:1198-1203.

8 O'Malley RL, Godoy G, Kanofosky JA, Taneja SS: The necessity of adrenalectomy at the time of radical nephrectomy: a systematic review. J Urol 2009;181:2009-2017.

9 Yap SA, Alibhai SM, Abouassaly R, Timilshina N, Margel D, Finelli A: Ipsilateral adrenalectomy at the time of radical nephrectomy impacts overall survival. BJU Int 2013; 111:E54-58.

10 Yap SA, Alibhai SM, Abouassaly R, Timilshina N, Finelli A: Do we continue to unnecessarily perform ipsilateral adrenalectomy at the time of radical nephrectomy? A population based study. J Urol 2012;187:398-404.

11 Deeks JJ: Systematic reviews of evaluations of diagnostic and screening tests; in: Egger M, Davey-Smith G, Altman D (eds): Systematic reviews in health care: meta-analysis in context. ed 2. London, BMJ Books, 2001, pp248-282.

12 Blakely S, Bratslavsky G, Zaytoun O, Daugherty M, Landas SK, Shapiro O: Preoperative cross-sectional imaging allows for avoidance of unnecessary adrenalectomy during RCC surgery. Urol Oncol 2015;33:22.e23-27.
13 Bekema HJ, MacLennan S, Imamura $\mathrm{M}$, Lam TB, Stewart F, Scott N, MacLennan G, McClinton S, Griffiths TR, Skolarikos A, MacLennan SJ, Sylvester R, Ljungberg B, N'Dow J: Systematic review of adrenalectomy and lymph node dissection in locally advanced renal cell carcinoma. Eur Urol 2013;64:799-810.

14 Lane BR, Tiong HY, Campbell SC, Fergany AF, Weight CJ, Larson BT, Novick AC, Flechner SM: Management of the adrenal gland during partial nephrectomy. J Urol 2009;181:2430-2436.

15 Bazzi WM, Sjoberg DD, Feuerstein MA, Maschino A, Verma S, Bernstein M, O’Brien MF, Jang T, Lowrance W, Motzer RJ, Russo $P$ : Long-term survival rates after resection for locally advanced kidney cancer: Memorial Sloan Kettering Cancer Center 1989 to 2012 experience. J Urol 2015;193:1911-1916.

16 Yokoyama H, Tanaka M: Incidence of adrenal involvement and assessing adrenal function in patients with renal cell carcinoma: Is ipsilateral adrenalectomy indispensable during radical nephrectomy? BJU Int 2005;95: 526-529.

17 Nakada T, Kubota Y, Sasagawa I, Yagisawa T, Watanabe M, Ishigooka M: Therapeutic outcome of primary aldosteronism: Adrenalectomy versus enucleation of aldosterone-producing adenoma. J Urol 1995;153: $1775-1780$. 